\title{
Shunting for normal pressure hydrocephalus in patients with neurodegenerative disorders
}

\author{
Joseph H. Friedman
}

Received: 12 April 2013/Revised: 29 April 2013/Accepted: 30 April 2013/Published online: 15 May 2013

(C) Springer-Verlag Berlin Heidelberg 2013

\section{Dear Sirs,}

The syndrome of idiopathic normal pressure hydrocephalus (iNPH) has been a murky subject since it was first described. Confounding understanding of iNPH has been the frequent comorbidities that accompany it [1]. The article by Magdalinou et al. [2] raises the issue of a less common confounding diagnosis, in particular progressive supranuclear palsy (PSP). This is a particularly pertinent concern since there are no reliable diagnostic tests for either condition. The diagnosis of iNPH cannot be confirmed by pathology and is, therefore, only "proven" when the patient has a clear and prolonged response to shunting, but there are no consensus criteria for defining what constitutes a meaningful response or how long the improvement should be maintained.

I have encountered three patients who were thought to have iNPH, having met the usual criteria for diagnosis, and having enjoyed dramatic improvement with ventricular peritoneal shunting, only to develop progressive neurological decline several months later. They were ultimately given clinical diagnoses of corticobasal syndrome, PSP and atypical Parkinsonism (type unknown), respectively. All patients are still alive and all were evaluated by me for the first time after their shunt response was lost. Family reports, as well as the referring neurologist and neurosurgeon notes, however, confirm a major benefit from the shunt, sustained for six months (CBD, age 82 at time of shunt), one year (PSP, age 68 at time of shunt), seven years

J. H. Friedman $(\bowtie)$

Movement Disorders Program, Butler Hospital, 345 Blackstone

Blvd, Providence, RI 02906, USA

e-mail: Joseph_Friedman@brown.edu followed by slow decline for eight years (atypical parkinsonism, age 62 at time of shunt).

The prolonged response to shunting suggests that iNPH is a non-specific syndrome that may be part of a clinically or pathologically identifiable neurologic disorder or might exist in isolation. Although these three patients lacked signs that would have pointed to the diagnosis of their neurodegenerative disease at the time of shunting, their response raises the question of whether shunting would have been a good treatment for them even had these diagnostic signs been present, since there are no known symptomatic treatments for these disorders. In each case, the family and patient was pleased with the shunt benefit and would have chosen to have it again, even knowing the correct diagnosis.

Conflicts of interest Lectures: Teva, General Electric, UCB; Consulting: Teva; Addex Pharm; UCB; Lundbeck; Roche; Research: MJFox; NIH: EMD Serono; Teva; Acadia; Schering Plough; Avid; Royalties: Demos Press.

Ethical standard All human studies have been approved by the appropriate ethics committee and have therefore been performed in accordance with the ethical standards and laid down in the 1964 Declaration of Helsinki.

\section{References}

1. Bech-Azeddine R et al (2007) Idiopathic normal-pressure hydrocephalus: clinical comorbidity, correlated with cerebral biopsy findings and outcome of cerebrospinal fluid shunting. J Neurol Neurosurg Psychiatry 78(2):157-161

2. Magdalinou NK et al (2013) Normal pressure hydrocephalus or progressive supranuclear palsy? A clinicopathological case series. J Neurol 260(4):1009-1013 